\title{
Low-Molecular-Weight Polyethyleneimine Grafted Polythiophene for Efficient siRNA Delivery
}

\author{
Pan He, ${ }^{1}$ Kyoji Hagiwara, ${ }_{1}^{1}$ Hui Chong, ${ }^{2}$ Hsiao-hua Yu, ${ }^{2,3}$ and Yoshihiro Ito ${ }^{1}$ \\ ${ }^{1}$ Nano Medical Engineering Laboratory, RIKEN, 2-1 Hirosawa, Wako, Saitama 351-0198, Japan \\ ${ }^{2}$ Responsive Organic Materials Laboratory, RIKEN, 2-1 Hirosawa, Wako, Saitama 351-0198, Japan \\ ${ }^{3}$ Institute of Chemistry, Academia Sinica, 128 Academic Road, Section 2, Nankang, Taipei 11529, Taiwan \\ Correspondence should be addressed to Hsiao-hua Yu; bruceyu@gate.sinica.edu.tw and Yoshihiro Ito; y-ito@riken.jp
}

Received 4 April 2015; Revised 13 June 2015; Accepted 23 June 2015

Academic Editor: Sanyog Jain

Copyright (C) 2015 Pan He et al. This is an open access article distributed under the Creative Commons Attribution License, which permits unrestricted use, distribution, and reproduction in any medium, provided the original work is properly cited.

\begin{abstract}
Owing to its hydrophilicity, negative charge, small size, and labile degradation by endogenous nucleases, small interfering RNA (siRNA) delivery must be achieved by a carrier system. In this study, cationic copolymers composed of low-molecular-weight polyethylenimine and polythiophenes were synthesized and evaluated as novel self-tracking siRNA delivery vectors. The concept underlying the design of these copolymers is that hydrophobicity and rigidity of polythiophenes should enhance the transport of siRNA across the cell membrane and endosomal membrane. A gel retardation assay showed that the nanosized complexes formed between the copolymers and siRNA were stable even at a molar ratio of $1: 2$. The high cellular uptake (>80\%) and localization of the copolymer vectors inside the cells were easily analyzed by tracking the fluorescence of polythiophene using fluorescent microscopy and cytometry. An in vitro luciferase knockdown (KD) assay in A549-luc cells demonstrated that the siRNA complexes with more hydrophobic copolymers achieved a higher $\mathrm{KD}$ efficiency of $52.8 \%$ without notable cytotoxicity, indicating protein-specific KD activity rather than solely the cytotoxicity of the materials. Our polythiophene copolymers should serve as novel, efficient, low cell toxicity, and label-free siRNA delivery systems.
\end{abstract}

\section{Introduction}

Since the pioneering work of RNA interference in 1998, gene suppression using small interfering RNA (siRNA) has received significant attention recently as an approach for treating inherited or acquired diseases [1]. However, finding an efficient drug delivery system (DDS) remains a major challenge for translating siRNA to the clinic [2]. Naked siRNA as unprotected oligonucleotides have a very short half-life in vivo (seconds to minutes) as a result of degradation by endogenous nucleases and rapid kidney filtration from circulation owing to their small size [3]. Following cellular internalization, the siRNA must also escape the endosome, because siRNA must enter the cytosol to have a therapeutic effect [4]. Thus, effective vehicles for siRNA delivery must demonstrate siRNA binding, low cytotoxicity, effective cellular uptake, and endosome escape and most importantly show evidence of siRNA-induced knockdown $[5,6]$.

Branched polyethylenimine (PEI) with a molecular weight of $25 \mathrm{kDa}$, named PEI-25K, and its derivatives have been the most popular cationic polymers for in vitro and in vivo gene delivery. This is because of their superior buffering capacity, which allows cargoes to escape the endosome to the cytoplasm by a hypothesized "proton sponge" mechanism $[7,8]$. However, high-molecular-weight PEI is still limited by cytotoxic issues, as assessed by the in vitro metabolic activity of cells [9]. Recently, low-molecular-weight PEIs with better biocompatibility, but low gene loading capacity, have proven to be valuable gene vectors after hydrophobic modification or cross-linking [10, 11]. Hydrophobic alkyl-modified PEI$2 \mathrm{k} /$ carbon-dot nanocomposites were found to be efficient for in vitro gene delivery with low cytotoxicity [12]. Thus, it is likely that there will be more in vitro and in vivo studies using hydrophobic-modified low-molecular-weight PEI for gene delivery [13].

Conjugated polymers such as polythiophenes [14] and poly(p-phenylene ethynylene) [15] and their nanoparticles [16] have emerged as novel gene delivery vectors, because of their potential cell-penetrating ability owing to their rigid chains, and such polymers are easy to use as traceable delivery 

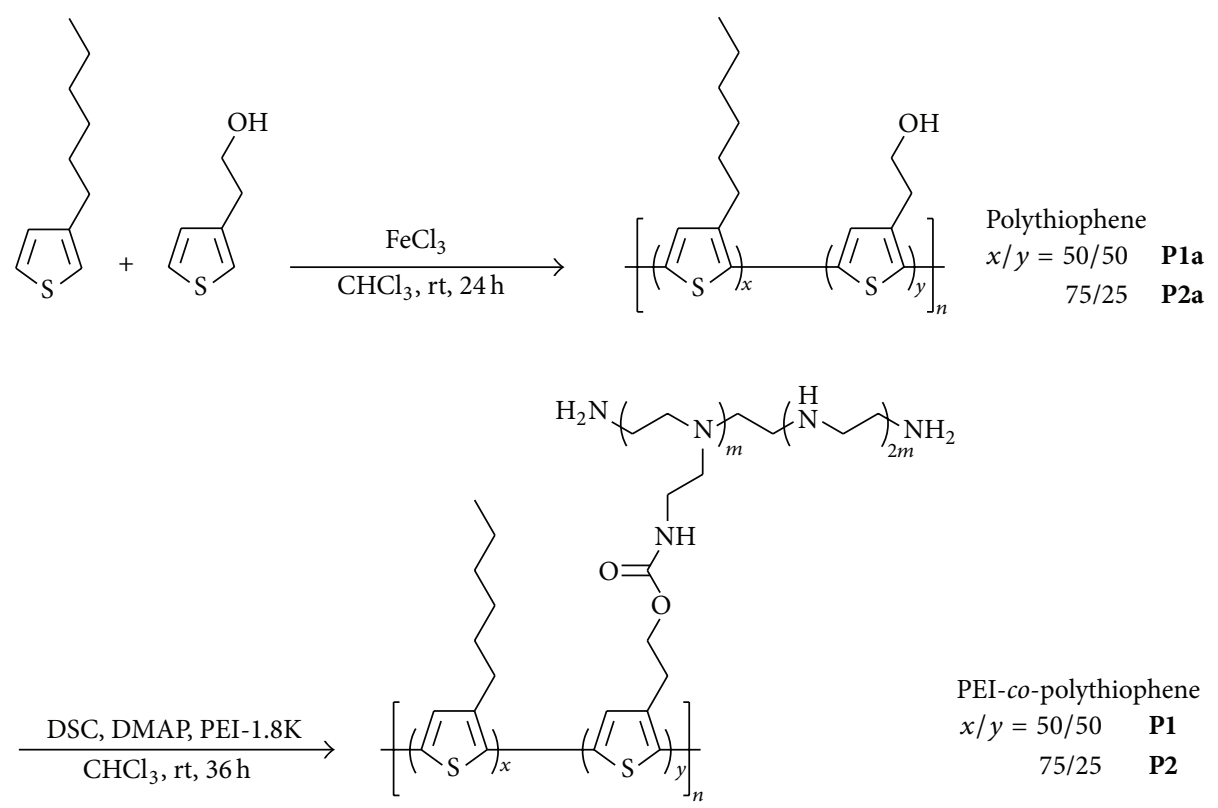

Scheme 1: Synthesis of PEI-co-polythiophenes copolymers P1 and P2 for siRNA delivery.

vehicles [17]. For example, monodispersed polyfluorene nanoparticles showed outstanding RNA-binding capacity and induced a knockdown efficiency of $23.9 \%$ with no significant cytotoxicity [18]. Jubeli et al. recently reported the potential of polyene-based cationic lipids as visually traceable siRNA transfer reagents for inhibition of luciferase expression [19]. To improve the knockdown or silencing efficiency is a challenge for conjugated polymeric gene carriers.

In our previous study, polyethylenedioxythiophenes with a cell-membrane-mimicking strategy were synthesized and showed specific neuron targeting and enhanced neuron cell adhesion and proliferation [20]. Similar to other conjugated polymers, polythiophenes may have possible cell-membrane penetrating ability via the rigid hydrophobic main chain. In this study, we designed copolymers composed of PEI-1.8K and hydrophobic polythiophenes (as shown in Scheme 1) as high-performance siRNA carriers. PEI-1.8K acted as a gene-condensing agent to form positively charged nanosized complexes with siRNA with minimal cytotoxicity. Moreover, polythiophenes with hydrophobic hexyl groups were employed both for fluorescence label-free function and for enhancing permeation across the cell membrane. The formation of the polymer/siRNA complex, cellular uptake and localization of fluorescence polymers, and a siRNA-mediated luciferase knockdown assay were carried out to evaluate this novel visualized siRNA vector.

\section{Materials and Methods}

2.1. Materials for Polymer Synthesis. Branched polyethylenimine (PEI-1.8K), anhydrous ferric chloride $\left(\mathrm{FeCl}_{3}\right)$, 4-(dimethylamino)pyridine (DMAP), and N, ${ }^{\prime}$-disuccinimidyl carbonate (DSC) were purchased from Wako Pure Chemical Industries, Ltd. (Tokyo, Japan) and were used without further purification. Dialysis membranes $\left(M_{\text {mwco }}=7,000 \mathrm{Da}\right)$ were obtained from Spectro Laboratories Inc. (Sylmar, CA, USA) and were used according to the manufacturer's instruction. 3-Hexylthiophene, 3-thiophene ethanol, and all other chemicals were from Tokyo Kasei Kogyo Co. Ltd. (TCI, Tokyo, Japan) and were used without further purification.

2.2. Cell Culture and siRNA. A549 cells stably expressing the luciferase gene (A549-luc) were grown in F-12K (Invitrogen, Life Technologies, Rockville, MD, USA) supplemented with $10 \%$ fetal bovine serum (FBS) and $200 \mu \mathrm{g} / \mathrm{mL}$ geneticin (Invitrogen). The cells were maintained at $37^{\circ} \mathrm{C}$ in a humidified atmosphere with $5 \% \mathrm{CO}_{2}$. All gene knockdown assays were performed by the siRNA against luciferase gene (sense strand: $5^{\prime}$-cuuAcGcuGaGuAcuucGAT ${ }^{*} \mathrm{~T}-3^{\prime}$ and antisense strand: $5^{\prime}$-UCGAAGUACUCAGCGUAAGT*T-3' [4]) and mismatch control siRNA, which was confirmed to have no significant knockdown activity against luciferase gene.

\subsection{Synthesis of Copolymers}

2.3.1. Synthesis of Poly(3-hexylthiophene-co-thiopheneethanol) P1a and P2a. P1a and P2a were synthesized by a previously reported, anhydrous $\mathrm{FeCl}_{3}$ catalyzed, chemical oxidative coupling approach with different feed ratios of the thiophene monomers [21]. A mixture of monomer solutions in $\mathrm{CHCl}_{3}$ was added to the suspension of anhydrous $\mathrm{FeCl}_{3}$ (4 equal moles of monomers) in $\mathrm{CHCl}_{3}$ under nitrogen and then stirred for $24 \mathrm{~h}$ at room temperature. After dedoping by ammonia water, the resulting fluorescent polythiophenes were purified by dissolving in $\mathrm{CHCl}_{3}$, precipitating in methanol, and then drying. The obtained P1a and P2a are dark red solids. 
2.3.2. Conjugation of PEI-1.8K. Pla was dissolved in dry chloroform ( $1 \mathrm{mM}$ pendent hydroxyl group) and then DMAP $(5 \mathrm{mM})$ and DSC $(5 \mathrm{mM})$ were added slowly under magnetic stirring. After $6 \mathrm{~h}$, a chloroform solution of PEI-1.8K (1 mM) was added to the reaction mixture and stirred for another $36 \mathrm{~h}$ [22]. The reaction mixture was concentrated and precipitated in hexane to remove overdosed DMAP and DSC. Excess PEI was removed by dialysis in methanol and then water for 3 days. The final copolymers were obtained as rust red solids after freeze-drying.

2.3.3. Characterization of Polymers. The structures of the synthesized compounds were identified by ${ }^{1} \mathrm{H}$ NMR spectroscopy (JEOL AL400, Tokyo, Japan). The weight-average molecular weight $\left(M_{\mathrm{w}}\right)$, number-average molecular weight $\left(M_{n}\right)$, and the distribution $\left(M_{\mathrm{w}} / M_{n}\right)$ of the polymers were measured on a Waters GPC system, which was equipped with a Waters 1515 HPLC solvent pump, a Waters 2414 refractive index detector, and two Waters Styragel high resolution columns, at $40^{\circ} \mathrm{C}$ using HPLC grade THF as eluent at a flow rate of $0.35 \mathrm{~mL} / \mathrm{min}$. Monodispersed polystyrenes were used to generate the calibration curve. Absorption spectra were measured using a JASCO V-550 UV/VIS spectrophotometer. Fluorescence spectra were measured using a JASCO FCT-133 spectrometer.

2.4. Preparation of Polymer/siRNA Complex. The polymer was dissolved in methanol and diluted with RNase-free water to make solutions of different concentrations (10 to $0.1 \mu \mathrm{M})$. The complexes were formed by gently mixing the siRNA solution with the polymer solution in equal volume and incubated for $30 \mathrm{~min}$ at room temperature. The final percentage of methanol in aqueous solution was below $5 \%$.

The particle size and zeta potential were determined by a zeta-potential and particles size analyzer (ELSZ-2PL, Otsuka Electronics Co. Ltd., Tokyo, Japan).

2.5. Gel Retardation Assay for siRNA. To determine whether our polymers could retard siRNA migration, various ratios of polymer in complex with siRNA $(1 \mu \mathrm{M})$ were prepared with different molar ratios (the molar ratio of the cationic polymers to RNA) and incubated at room temperature for $30 \mathrm{~min}$. Then the complex was mixed with loading buffer and applied to a $20 \%$ gel (Biocraft Co. Ltd., Tokyo, Japan), and electrophoresis was carried out for 60 min under a constant voltage of $100 \mathrm{~V}$. The gel was stained with ethidium bromide for $20 \mathrm{~min}$. After washing three times with water, the siRNA band was detected by a UV transmitter (ATTO Corporation, Tokyo, Japan). Two different loading buffers were used in this study. Loading buffer 1 contains glycerol (10\%, v/v), $5 \mathrm{mM}$ HEPES buffer ( $\mathrm{pH} 7.3$ ), and $1 \mathrm{mM}$ EDTA. Loading buffer 2 has two more components: Triton X-100 (1\%) and 60\% of potassium polyvinyl sulfate (Wako Pure Chemical Industries, Ltd., Tokyo, Japan) to disrupt the polymer/siRNA complex.

2.6. Cellular Uptake by Monitoring the Fluorescence of Polythiophene. Next, $2 \times 10^{5}$ cells (A549-luc) were cultured in a 12 -well plate in F-12K with $200 \mu \mathrm{g} / \mathrm{mL}$ geneticin. After $24 \mathrm{~h}$, the polythiophene solution was added to the cell culture medium (final concentration was $0.5 \mu \mathrm{M}$ ) and incubated for $72 \mathrm{~h}$. The uptake was directly monitored by the fluorescence from polythiophene using a fluorescence microscope (Zeiss, LSM 510 Meta, Jena, Germany) and the intensity was measured by a Tali image-based cytometer (Life Technologies).

2.7. Cellular Localization of Polymers. Then, $2 \times 10^{5}$ cells (A549-luc) were cultured on a glass bottom plate in F$12 \mathrm{~K}$ with $200 \mu \mathrm{g} / \mathrm{mL}$ geneticin. After $24 \mathrm{~h}$, the $0.5 \mathrm{mM}$ polythiophene solution was added to the cell culture medium (final concentration was $0.25 \mu \mathrm{M}$ ) and incubated for $48 \mathrm{~h}$. Confocal microscopy was performed on a Nikon Eclipse TiE inverted confocal fluorescence microscope (Nikon Instruments, Tokyo, Japan) using 60x oil immersion Plan Apo VC and 1.4-numerical aperture objective. Samples were excited with 488 and $561 \mathrm{~nm}$ solid-state lasers, and the emission was captured with a Nikon C2 confocal scan head (Nikon) interfaced to a PC running NIS-Elements $\mathrm{C}$ software. Threedimensional stacks were generated from a series of confocal plane images with $1.0 \mu \mathrm{m}$ steps.

2.8. Knockdown Assay and Cytotoxicity Assessment. A549luc cells were seeded at a density of $6 \times 10^{3}$ cells/well in fresh F12-K medium containing 10\% FBS without geneticin and incubated for $24 \mathrm{~h}$. The polymer/siRNA complexes with different molar ratios were added to the culture medium, gently mixed, and then incubated for $72 \mathrm{~h}$. As a positive control, cells were transfected with DharmaFECT1 transfection reagent (GE Healthcare, Lafayette, CO, USA)/siRNA complexes that were prepared according to the manufacturer's instructions. After $72 \mathrm{~h}, 100 \mu \mathrm{L}$ of the PicaGene LT2.0 luminescence reagent (Toyo Inki, Tokyo, Japan) was added to the cells and the luciferase activities were analyzed using a Multimode Plate Reader (EnSpire, PerkinElmer), according to the manufacturer's protocol. Cell viability was determined using the CellTiter-Glo kit (Promega, Madison, WI, USA), according to the manufacturer's protocol. The viability of nontreated control cells was arbitrarily defined as $100 \%$.

\section{Results and Discussion}

3.1. Synthesis of PEI-co-polythiophenes Copolymers. Scheme 1 describes the synthetic route of the copolymers. Initially, polythiophenes P1a and P2a were obtained by oxidation coupling at monomer feed ratios of $50: 50$ and $75: 25$, respectively. We further grafted PEI onto the hydrophobic polythiophenes by reacting DSC activated hydroxyl groups on polythiophenes with the primary amines on PEI. The final copolymers were named $\mathbf{P 1}$ and $\mathbf{P} 2$ for convenience.

The ${ }^{1} \mathrm{H}-\mathrm{NMR}$ spectra in Figure 1 showed that the peaks from 0.9 to $1.7 \mathrm{ppm}$ represent the protons on the alkyl side chain $\left(-\mathrm{CH}_{3}\right.$ and $\left.-\mathrm{CH}_{2}-\right)$ of P2a. The weak signal at $7.0 \mathrm{ppm}$ corresponds to the proton of the end-capped thiophene ring. The average molecular weights of P1a and P2a are $20 \mathrm{~kg} \mathrm{~mol}^{-1}\left(M_{\mathrm{w}} / M_{n} 1.85\right)$ and $32 \mathrm{~kg} \mathrm{~mol}^{-1}\left(M_{\mathrm{w}} / M_{n} 3.88\right)$, respectively (data not shown). After PEI conjugation to the side chain, the solubility of the copolymers significantly 


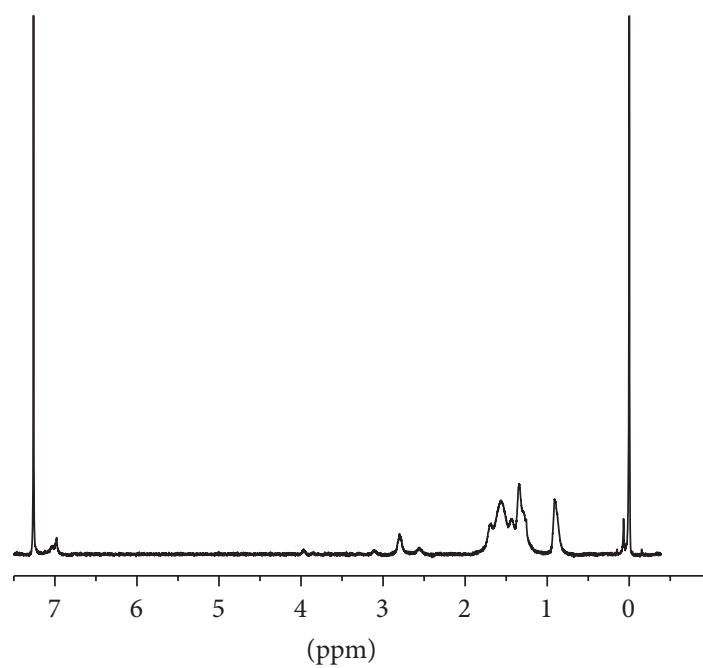

- P2a

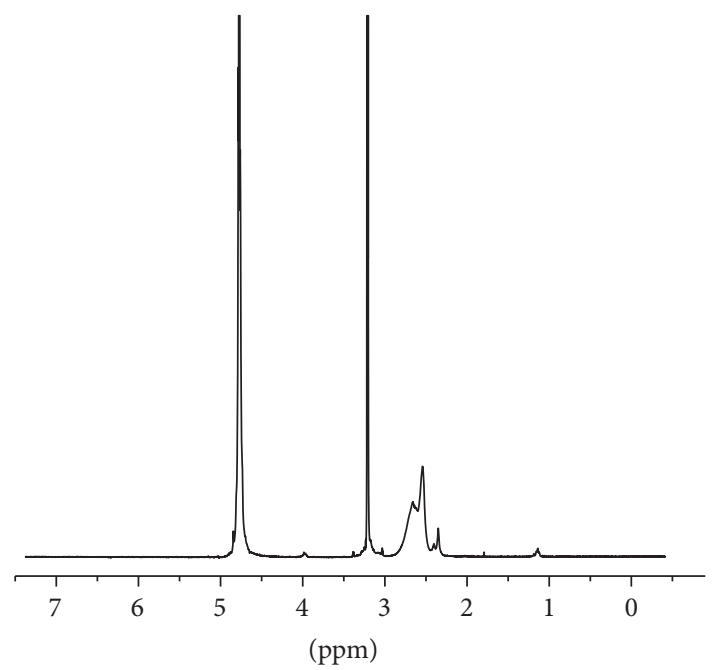

$-\mathbf{P 2}$

(a)

(b)

Figure 1: ${ }^{1} \mathrm{H}$-NMR spectra of polythiophenes before (P2a in $\left.\mathrm{CDCl}_{3}\right)$ and after PEI conjugation (P2 in $d$-methanol).

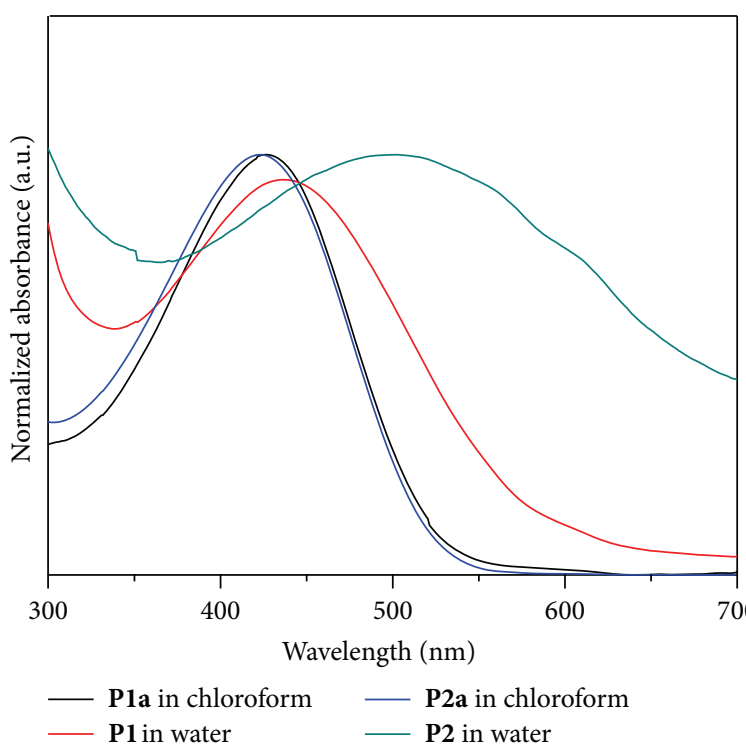

(a)

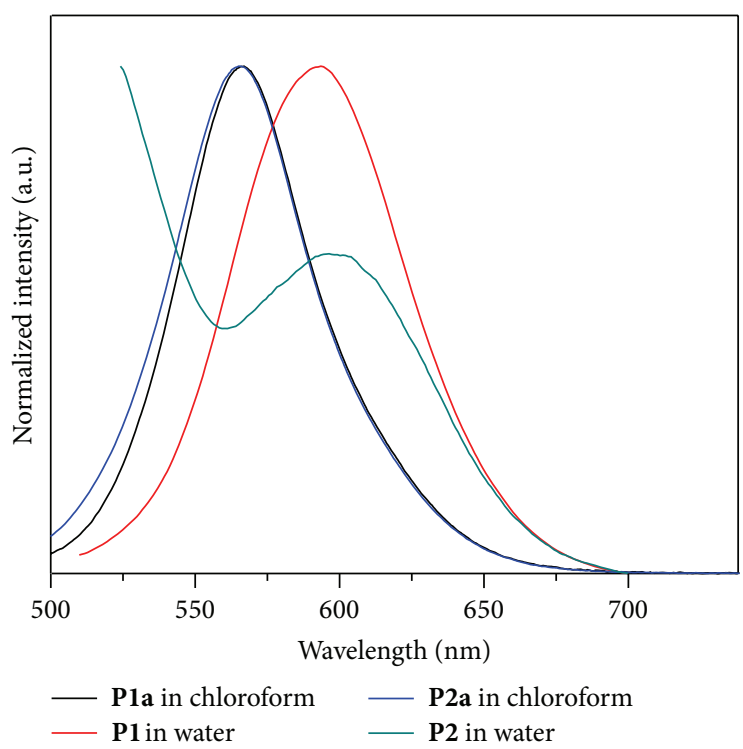

(b)

Figure 2: (a) UV-Vis absorption and (b) fluorescence spectra of copolymers in chloroform or in aqueous solution at room temperature. The value has been normalized.

changed. P1 and P2 only dissolve in methanol and water and swell in chloroform. The ${ }^{1} \mathrm{H}$ NMR spectrum of $\mathbf{P 2}$ in $d$-methanol showed strong signals at $\sim 2.5 \mathrm{ppm}$ (Figure 1), which represents the protons of PEI-1.8K. Unfortunately, we did not obtain the GPC data of $\mathbf{P 1}$ and $\mathbf{P} \mathbf{2}$ because of poor solubility. Compared with a reported multistep approach involving a complicated fabrication of a conjugated polymer, the protocol presented herein is superior because it is simple and time effective.

3.2. UV-Vis and Fluorescence Spectra of Copolymers. Pla and P2a have absorption bands around $425 \mathrm{~nm}$ (Figure 2(a)), which are attributed to the coil conformation of polythiophenes in solution [23]. After conjugation with PEI, there is a slight red shift $(436 \mathrm{~nm})$ of the UV-absorption spectrum of P1. Surprisingly, with a lower PEI graft density (25\%), the copolymer $\mathbf{P} 2$ showed a broad absorption band at $\lambda=$ $500 \mathrm{~nm}$ (Figure 2(a)), $70 \mathrm{~nm}$ higher than that observed for P2a. This is probably because of the formation of a selfassembly of $\mathbf{P 2}$ in water displaying main chain aggregation. Charged PEI is expected to stretch as the hydrophilic shell, whereas the hydrophobic polyalkylthiophene functions as the core (Scheme 2). This process is associated with an aggregated chromophore backbone and a higher coplanarity. 


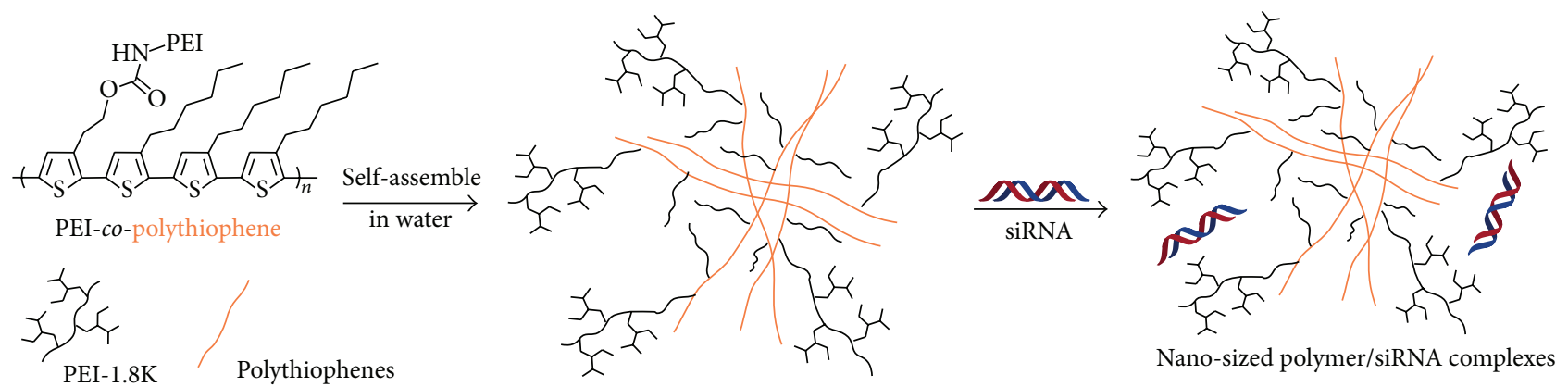

Scheme 2: Demonstration of the self-assembly of copolymers in water and the formation of their nanosized complexes with siRNA.

Therefore, this process leads to an increase in the conjugation length and a red shift of the UV-Vis absorption. As for the fluorescence properties, there is a similar wavelength maximum at $560 \mathrm{~nm}$ before PEI conjugation and at $590 \mathrm{~nm}$ for the PEI-conjugated graft copolymers (Figure 2(b)), which are easy to track by confocal microscopy at suitable excitation and emission ranges. It should be noted that the emission of $\mathbf{P 2}$ was considerably less intense than that of $\mathbf{P 2 a}$, presumably because of a fluorescence quenching by the aggregation of the conjugated main chains.

3.3. Formation of Polymer/siRNA Complexes. The binding capacity of siRNA with our cationic fluorescent copolymers was evaluated using a gel retardation assay at various molar ratios. As shown in Figure 3, the extent of retardation increases with the increasing ratio of $\mathbf{P 2} /$ siRNA. By association with loading buffer 1 , no obvious migrated siRNA bands were observed in the lanes where the molar ratio was larger than 1:2, indicating very strong binding by the $\mathbf{P} 2$ polymer. Even with the disruption effect of loading buffer 2 , which contains potassium polyvinyl sulfate to disrupt the polymer/siRNA complex, the migrating band became very weak at a 5:1 ratio and siRNA was still fully retarded by $\mathbf{P 2}$ at a molar ratio of $10: 1$. For the P1/siRNA complex, the bands were slightly weaker at the same molar ratios (data not shown). Therefore, we assume that $\mathbf{P 1}$ has slightly stronger siRNA binding capacity than $\mathbf{P} 2$, which is probably attributed to the higher PEI grafting density.

Although the siRNA complexes have a similar zeta potential of $\sim+30 \mathrm{mV}$, the complex sizes are quite different for the two polymers. As shown in Figure 4(a), P1/siRNA complexes were almost monodispersed nanoparticles with diameters of $184.9 \pm 62.5 \mathrm{~nm}$ and a narrow polydispersity index (PDI, 0.121). In contrast, polymer $\mathbf{P 2}$ formed a larger size of complex with siRNA, with a broader PDI of 0.241 at the same molar ratio of $5: 1$. The lower molecular weight and better water solubility of $\mathbf{P 1}$ may contribute to the smaller size and narrower distribution of their siRNA complexes. The diameter of the complex is a little smaller than the pure polymer nanoparticles in water. For example, the average sizes of $\mathbf{P 1}$ and $\mathbf{P 2}$ nanoparticles were determined to be $117.1 \pm 26.3$ and $208.0 \pm 46.7 \mathrm{~nm}$, which decreased to $104.1 \pm$ 24.8 and $184.2 \pm 46.3 \mathrm{~nm}$ after binding with siRNA at a $5: 1$ ratio (Figure $4(\mathrm{~b})$ ). This may be attributed to the slight

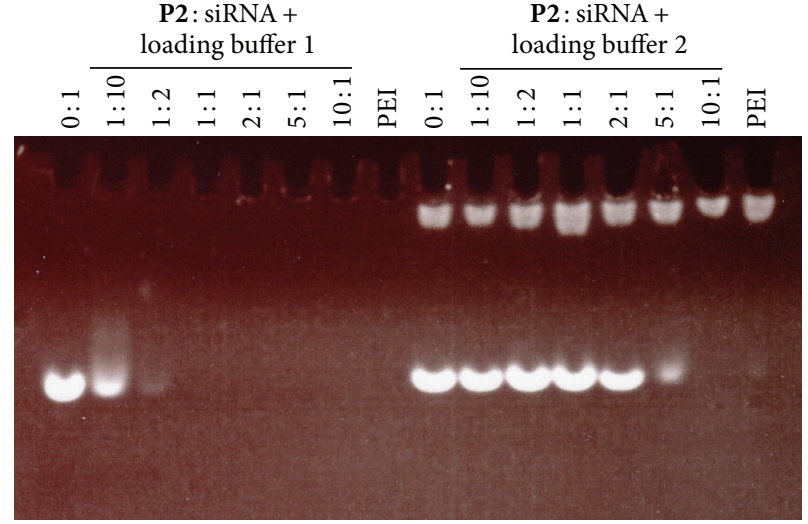

Figure 3: Copolymer P2 forms complexes with siRNA at different molar ratios and the binding stability was tested by gel electrophoresis. Loading buffer 1 contains glycerol, HEPES buffer ( $\mathrm{pH} 7.3$ ), and EDTA. Loading buffer 2 has two more components: Triton X-100 $(1 \% \mathrm{v} / \mathrm{v})$ and potassium polyvinyl sulfate (PVSK) to disrupt the polymer/siRNA complex.

condensation of ionized PEI by the negatively charged siRNA (Scheme 2). We investigated the cellular uptake and distribution of the fluorescent copolymer nanoparticles in the next step.

3.4. Cellular Uptake and Localization of Polythiophenes. Initially, the cellular uptake efficiency of polythiophenes was analyzed using fluorescence microscopy and Tali imagebased cytometry. As shown in Figure 5(a), clear green and weak red fluorescence from $\mathbf{P 1}$ were observed in the cytoplasm, but not in the nucleus of the cells, suggesting that uptake of $\mathbf{P 1}$ by the cells was successful. By using an imagebased cytometer, the uptake efficiency of $\mathbf{P 1}$ was $81 \%$ (by green fluorescence at $466 \mathrm{~nm}$ ) or $63 \%$ (by red fluorescence at $543 \mathrm{~nm}$ ) (Figure 5(b)). Similar cellular uptake $(\sim 88 \%)$ was observed using the P2/Alexa647-labeled siRNA complex (data not shown), indicating good cell-penetrating activity of polythiophene-based copolymers.

Next, to identify the detailed location of polythiophenes in the delivery process, P1 was added to the A549 cells and localization was determined by three-dimensional stacks generated from a series of confocal plane images with $1.0 \mu \mathrm{m}$ 


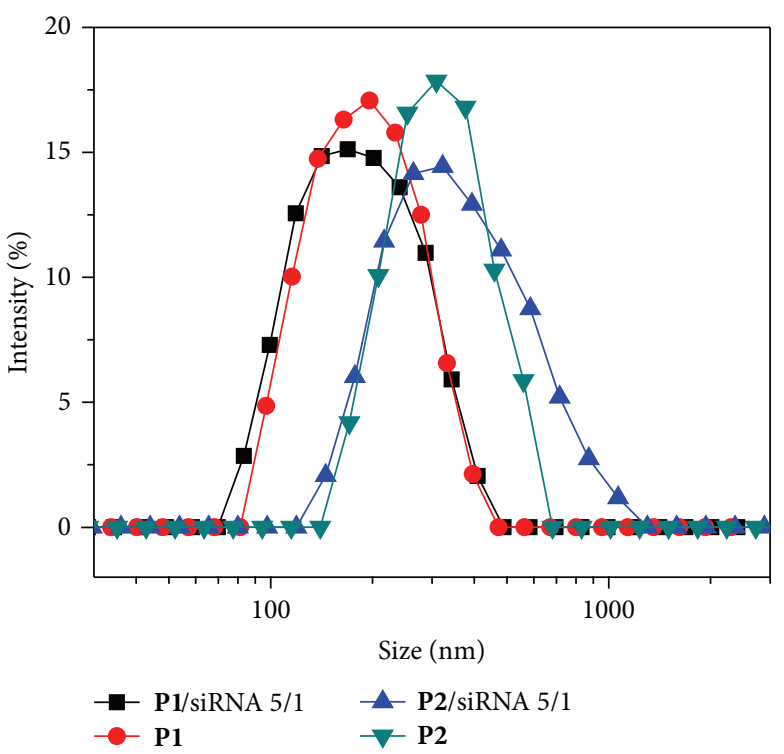

(a)

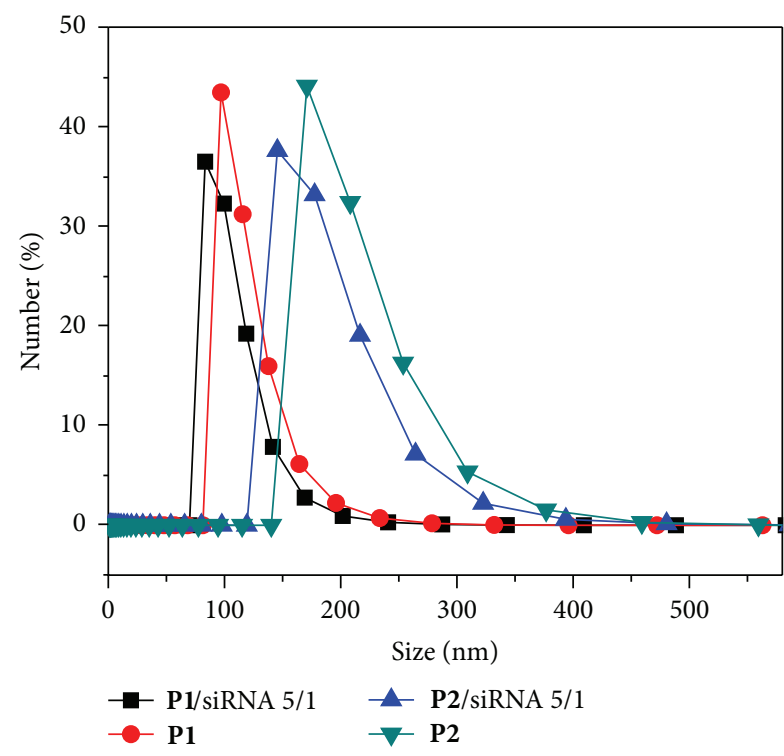

(b)

FIGURE 4: Particle size of polymer/siRNA complexes in water by DLS calculated by intensity (a) or number (b) average hydrodynamic diameters.

steps. According to Figure 5(c), a robust orange fluorescence was detected with a well-uniformed distribution in the cytoplasm. This indicated that $\mathbf{P 1}$ was able to internalize into cells and escape endosomes to localize in the cytosol. These results clearly proved that our copolymers are very promising agents as intracellular delivery systems.

3.5. In Vitro Knockdown and Cytotoxicity Evaluation. The goal of siRNA-based therapy is to knockdown the expression of a specific protein to achieve a specific therapeutic effect. Therefore, finally in this study, the luciferase gene-specific siRNA was chosen to evaluate the gene knockdown in A549luc cells. DharmaFECT1, an efficient commercial transfection reagent, was used as positive control and showed the best luciferase knockdown efficiency ( 80\%). As for plain PEI polymer, there was very weak knockdown activity $(<8.1 \%)$ and no obvious cytotoxicity when siRNA was applied with PEI-1.8K at different weight ratios (Figure 6(a)). This result corroborates with the previous report by Tian et al. and Yang et al. that low-molecular-weight PEIs have better biocompatibility but poor efficiency $[10,11]$. After hydrophobic modification by Pla, the percentage knockdown of the luciferase gene was enhanced up to $41.6 \%$ at the $0.50 \mathrm{mg} / \mathrm{mL}$ P1 complex with $10 \mathrm{nM}$ siRNA. The corresponding cell viability of A549luc was $72.3 \%$. At a mid-dose of $0.25 \mathrm{mg} / \mathrm{mL}$, the P1/siRNA complex showed $13.7 \% \mathrm{KD}$ efficiency with cell viability of 94.8\% (Figure 6(b)).

As for $\mathbf{P 2}$ with more hydrophobic alkyl chain content, up to $52.8 \%$ luciferase knockdown was achieved with a single P2/siRNA treatment without obvious cytotoxicity (88\% cell viability, Figures 7(a) and 7(b)), indicating protein-specific KD activity, but not because of the cytotoxicity of the materials. At a high molar ratio of $5: 1$, the P1/siRNA complex demonstrated a very high $\mathrm{KD}$ of $72.3 \%$; however, the cell viability decreased to $68.6 \%$. These results confirmed the effectiveness of our PEI-co-polythiophenes for gene delivery.

As gene silencing takes place at the mRNA stage in the cytosol, the fate of the DDS inside cells and intracellular localization must ensure the availability of siRNA in this compartment. In our design, PEI-1.8K should be the active compound to escape the endosomes. However, with the same RNA-binding capacity and surface positive charge, the P1/siRNA complexes with higher PEI density and smaller particle size showed much lower KD efficiency than hydrophobic P2. This implies that the hydrophobicity of the alkyl side chain also plays an important role in the effective delivery of siRNA.

We hypothesize here two possible factors that may explain the high uptake and good localization of our polymeric gene vectors. The first factor involves binding of the hydrophobic alkyl chain to the inner lipid bilayers of the cell membrane, thereby contributing to the high cellular uptake. The other factor is that the rigid polythiophene backbone may help the compound to penetrate the cell and other membranes, thus promoting internalization of DDS and also endosome escape. These two factors may define the higher performance of P2 compared to the more hydrophilic P1. Further studies are required to clarify whether these two factors are responsible for the high uptake and knockdown efficiency. Finally, because siRNA delivery is an intricate and complicated multiple-step process, the effective tracking of our DDS is important for providing feedback to the optimization of carrier design and delivery efficiency [24].

\section{Conclusions}

Cationic and fluorescent copolymers based on PEI and polythiophenes have been designed and synthesized for 

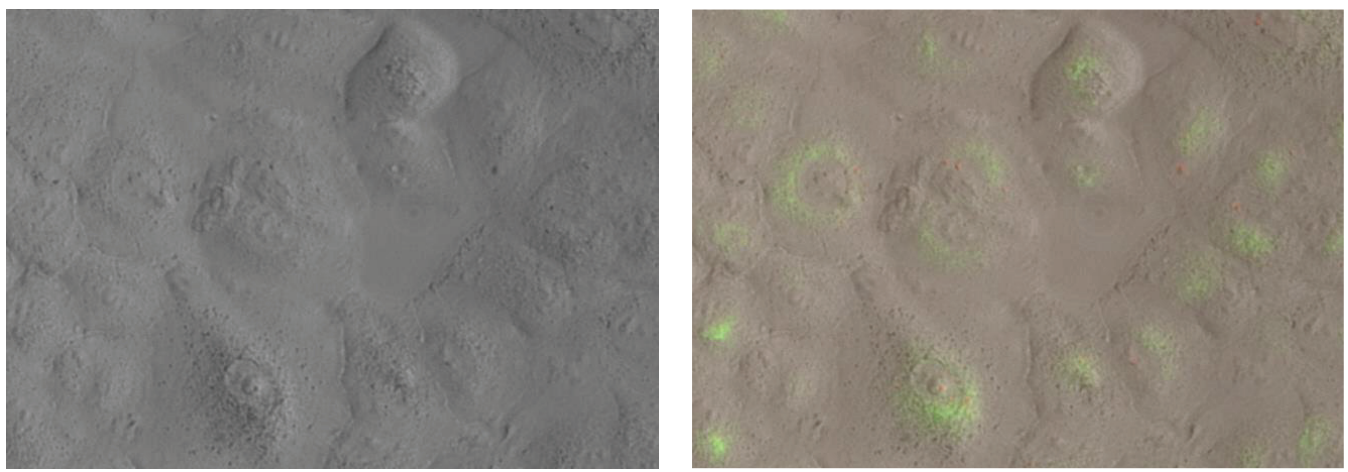

(a)
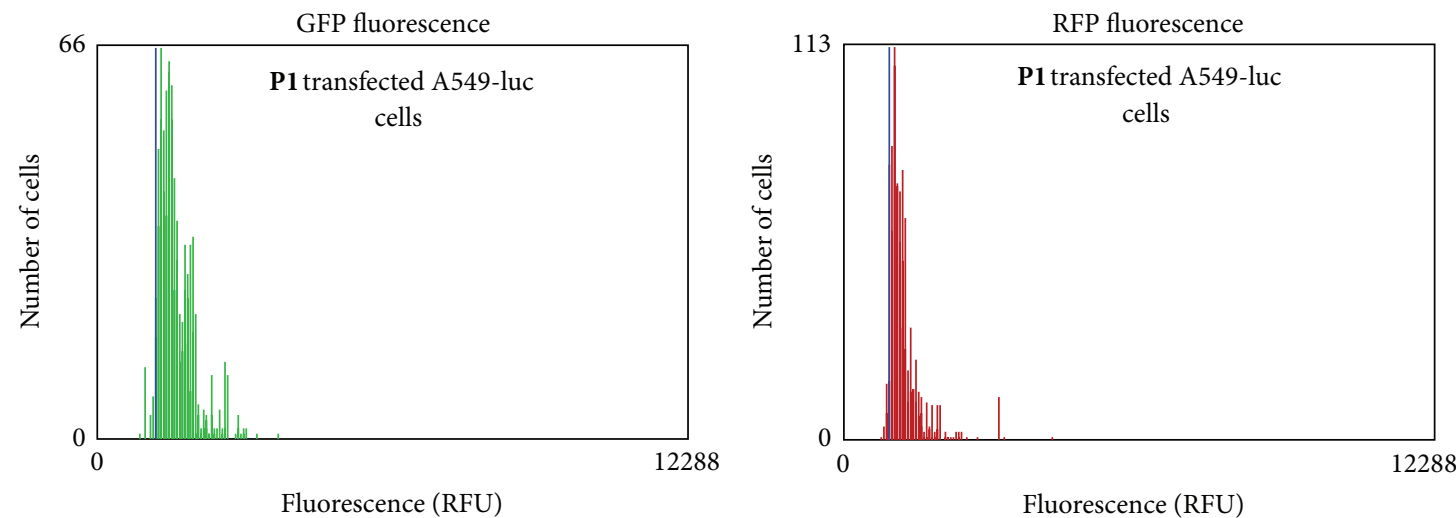

(b)
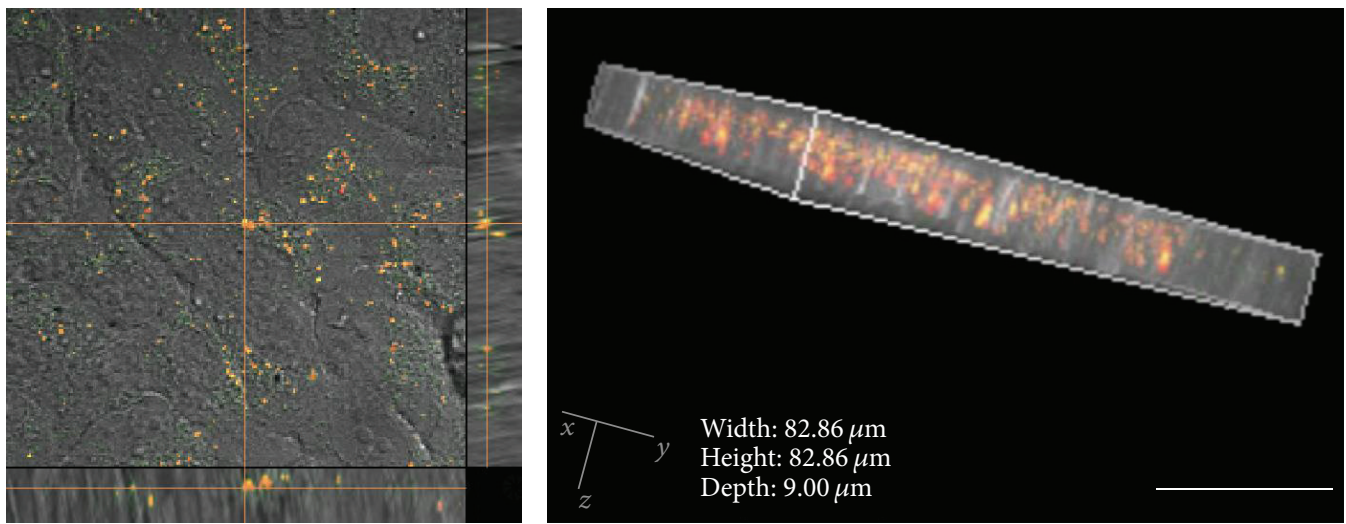

(c)

FIGURE 5: Copolymer P1 (0.5 $\mu \mathrm{M})$ was transfected to the A549-luc cells for $72 \mathrm{~h}$. (a) Fluorescence was detected by a fluorescent microscope, (b) determination of cellular uptake by a Tali image-based cytometer, (c) monitoring the localization of P1 by three-dimensional stacks generated from a series of confocal plane images with $1.0 \mu \mathrm{m}$ steps.

siRNA delivery. The conjugated polythiophene endows the copolymer, as the DDS, with a label-free advantage, which was found to show good distribution inside A549 cells. The low-molecular-weight PEI-1.8K allows the graft copolymer to form nanosized stable complexes with siRNA with low cytotoxicity. Significant knockdown of the targeted protein expression was achieved by siRNA delivered through both P1 and $\mathbf{P} 2$ copolymers at an appropriate dosage. The hydrophobicity of both the thiophene main chain and the alkyl side chain may also contribute to the cellular uptake and drug delivery performance. In conclusion, this study demonstrated that PEI-co-polythiophenes copolymers might serve as novel, efficient, low toxic, and self-tracking siRNAdelivery vectors. We will focus on the function of hydrophobic interactions and the possible endosome escape mechanism of the fluorescent copolymers for gene delivery in future work.

\section{Conflict of Interests}

The authors report no conflict of interests. 


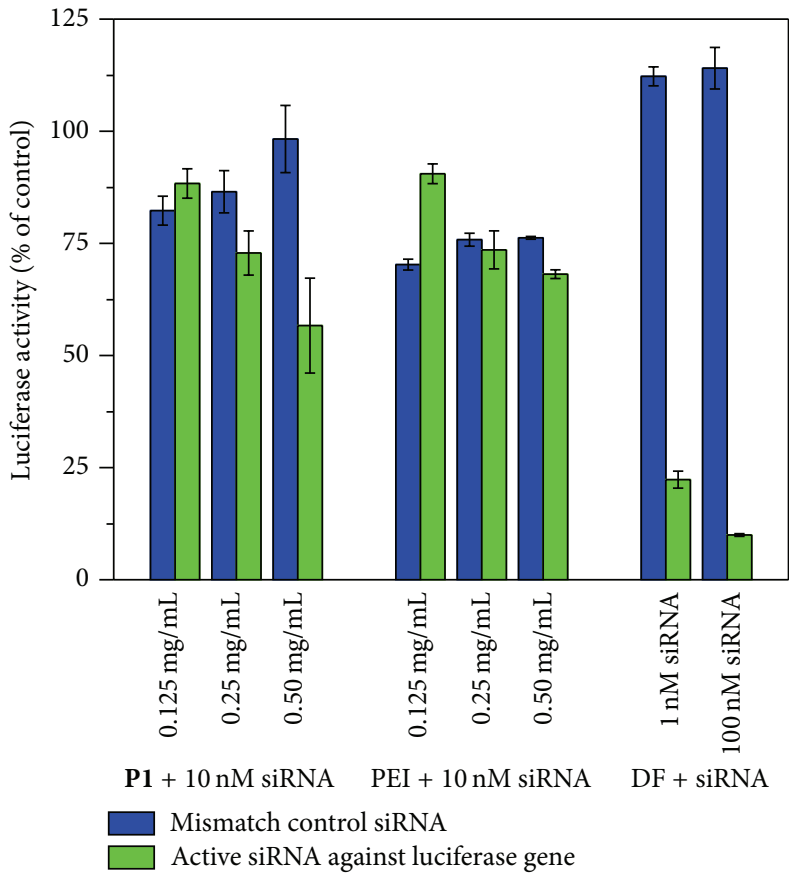

(a)

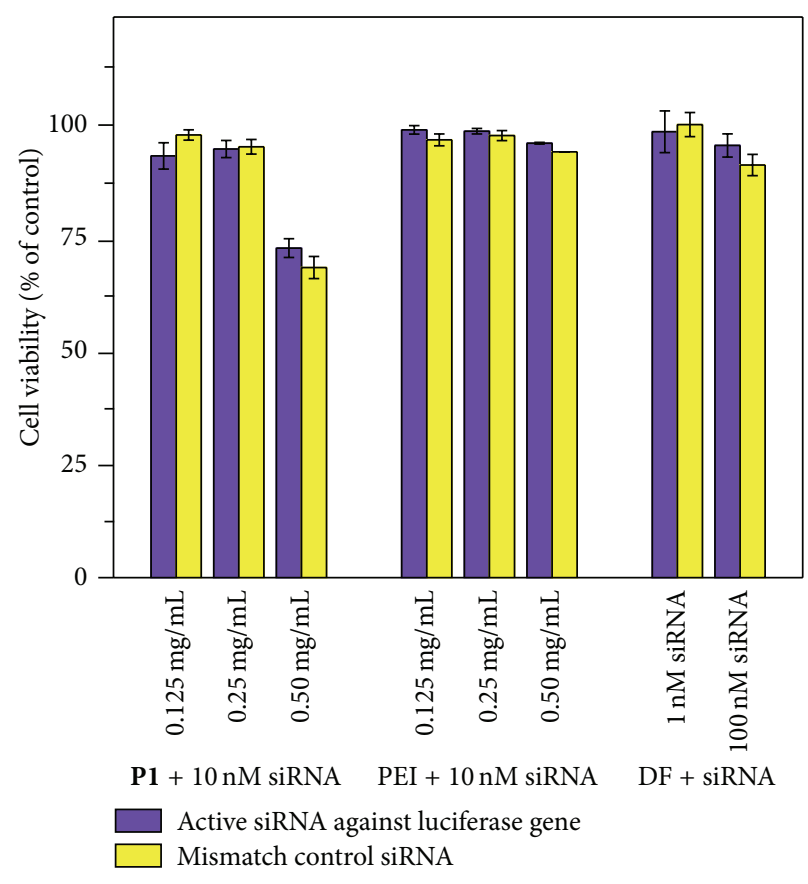

(b)

FIGURE 6: In vitro gene knockdown and cytotoxicity results of P1/siRNA and PEI-1.8K/siRNA complexes in A549-luc cells. (a) Relative expression of luciferase after $72 \mathrm{~h}$ incubation. (b) Cell viability determined using the CellTiter-Glo kit. The concentration of siRNA was fixed at $10 \mathrm{nM}$ and DharmaFECT1 (DF) was used as a positive control carrier.

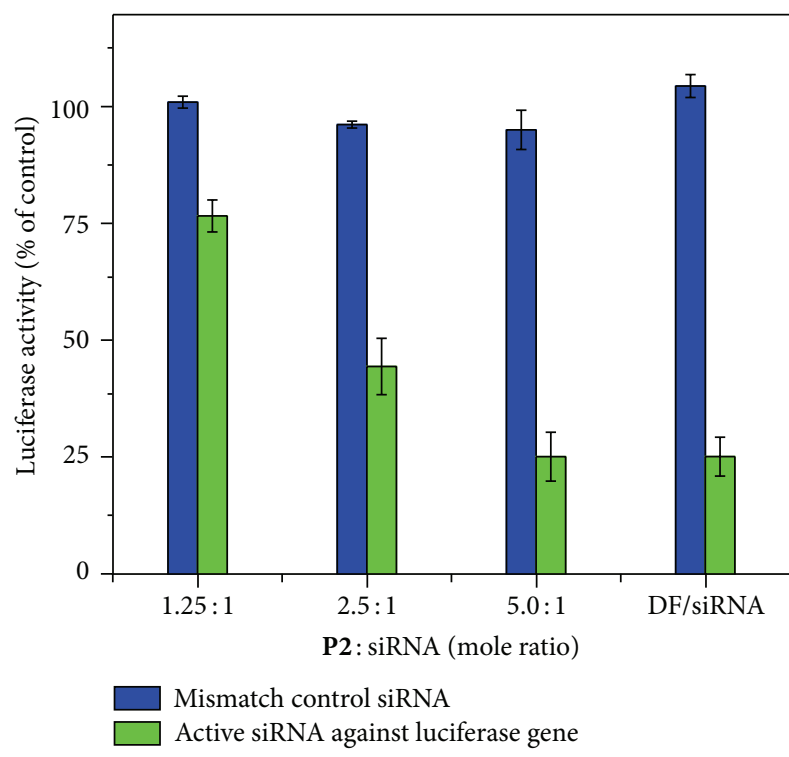

(a)

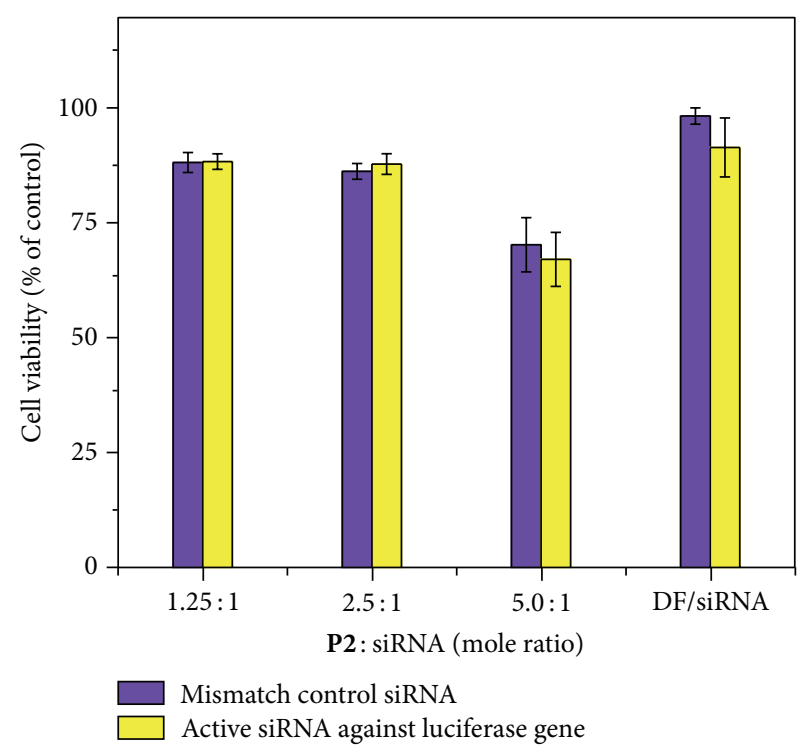

(b)

FIGURE 7: In vitro gene knockdown and cytotoxicity results of P2/siRNA complexes in A549-luc cells. (a) Relative expression of luciferase after $72 \mathrm{~h}$ incubation. (b) Cell viability determined using the CellTiter-Glo kit. The concentration of siRNA is fixed at $10 \mathrm{nM}$ and DharmaFECT1 (DF) was used as a positive control carrier. 


\section{Authors' Contribution}

Pan He and Kyoji Hagiwara contributed equally to this work.

\section{Acknowledgments}

This work was financially supported by Takeda Pharmaceutical Company Ltd. The authors thank Drs. Watanabe and Kawamata at Takeda Pharmaceutical Company Ltd. and Dr. Haichao Zhao at the Responsive Organic Materials Laboratory of RIKEN for helpful discussions. They thank Dr. Masuda at Takeda Pharmaceutical Company Ltd. for his help in the knockdown assay.

\section{References}

[1] A. Fire, S. Xu, M. K. Montgomery, S. A. Kostas, S. E. Driver, and C. C. Mello, "Potent and specific genetic interference by doublestranded RNA in Caenorhabditis elegans," Nature, vol. 391, no. 6669, pp. 806-811, 1998.

[2] N. P. Gabrielson, H. Lu, L. Yin, K. H. Kim, and J. Cheng, "A cellpenetrating helical polymer for siRNA delivery to mammalian cells," Molecular Therapy, vol. 20, no. 8, pp. 1599-1609, 2012.

[3] D. C. Forbes and N. A. Peppas, "Polycationic nanoparticles for siRNA delivery: comparing ARGET ATRP and UV-initiated formulations," ACS Nano, vol. 8, no. 3, pp. 2908-2917, 2014.

[4] R. U. Svensson, M. R. Shey, Z. K. Ballas et al., "Assessing siRNA pharmacodynamics in a luciferase-expressing mouse," Molecular Therapy, vol. 16, no. 12, pp. 1995-2001, 2008.

[5] R. Qi, S. Liu, J. Chen et al., "Biodegradable copolymers with identical cationic segments and their performance in siRNA delivery," Journal of Controlled Release, vol. 159, no. 2, pp. 251260, 2012.

[6] T. Fröhlich, D. Edinger, V. Russ, and E. Wagner, "Stabilization of polyplexes via polymer crosslinking for efficient siRNA delivery," European Journal of Pharmaceutical Sciences, vol. 47, no. 5, pp. 914-920, 2012.

[7] J. Intra and A. K. Salem, "Characterization of the transgene expression generated by branched and linear polyethylenimineplasmid DNA nanoparticles in vitro and after intraperitoneal injection in vivo," Journal of Controlled Release, vol. 130, no. 2, pp. 129-138, 2008.

[8] T. Feng, X. Dong, H. Tian et al., "PEGylated poly(aspartate-gOEI) copolymers for effective and prolonged gene transfection," Journal of Materials Chemistry B, vol. 2, no. 18, pp. 2725-2732, 2014.

[9] L. Parhamifar, A. K. Larsen, A. C. Hunter, T. L. Andresen, and S. M. Moghimi, "Polycation cytotoxicity: a delicate matter for nucleic acid therapy-focus on polyethylenimine," Soft Matter, vol. 6, no. 17, pp. 4001-4009, 2010.

[10] P. Y. Teo, C. Yang, J. L. Hedrick et al., "Hydrophobic modification of low molecular weight polyethylenimine for improved gene transfection," Biomaterials, vol. 34, no. 32, pp. 7971-7979, 2013.

[11] H. Tian, W. Xiong, J. Wei et al., "Gene transfection of hyperbranched PEI grafted by hydrophobic amino acid segment PBLG," Biomaterials, vol. 28, no. 18, pp. 2899-2907, 2007.

[12] L. Wang, X. Wang, A. Bhirde et al., "Carbon-dot-based twophoton visible nanocarriers for safe and highly efficient delivery of siRNA and DNA," Advanced Healthcare Materials, vol. 3, no. 8, pp. 1203-1209, 2014.
[13] H. Tian, Z. Guo, J. Chen et al., "PEI conjugated gold nanoparticles: efficient gene carriers with visible fluorescence," Advanced Healthcare Materials, vol. 1, no. 3, pp. 337-341, 2012.

[14] A. C. Carreon, W. L. Santos, J. B. Matson, and R. C. So, "Cationic polythiophenes as responsive DNA-binding polymers," Polymer Chemistry, vol. 5, no. 2, pp. 314-317, 2014.

[15] L. Zhang, Q.-H. Yin, J.-M. Li, H.-Y. Huang, Q. Wu, and Z.-W. Mao, "Functionalization of dendritic polyethylene with cationic poly(p-phenylene ethynylene) enables efficient siRNA delivery for gene silencing," Journal of Materials Chemistry B, vol. 1, no. 17, pp. 2245-2251, 2013.

[16] J. H. Moon, E. Mendez, Y. Kim, and A. Kaur, "Conjugated polymer nanoparticles for small interfering RNA delivery," Chemical Communications, vol. 47, no. 29, pp. 8370-8372, 2011.

[17] L. Zhang, Q. Yin, H. Huang, and B. Wang, "Conjugation of cationic poly(p-phenylene ethynylene) with dendritic polyethylene enables live-cell imaging," Journal of Materials Chemistry B, vol. 1, no. 6, pp. 756-761, 2013.

[18] R. Jiang, X. Lu, M. Yang, W. Deng, Q. Fan, and W. Huang, "Monodispersed brush-like conjugated polyelectrolyte nanoparticles with efficient and visualized SiRNA delivery for gene silencing," Biomacromolecules, vol. 14, no. 10, pp. 36433652, 2013.

[19] E. Jubeli, L. Raju, N. A. Khalique et al., "Polyene-based cationic lipids as visually traceable siRNA transfer reagents," European Journal of Pharmaceutics and Biopharmaceutics, vol. 89, pp. 280-289, 2015.

[20] B. Zhu, S.-C. Luo, H. Zhao et al., "Large enhancement in neurite outgrowth on a cell membrane-mimicking conducting polymer," Nature Communications, vol. 5, article 4523, 2014.

[21] B. Kim, L. Chen, J. Gong, and Y. Osada, "Titration behavior and spectral transitions of water-soluble polythiophene carboxylic acids," Macromolecules, vol. 32, no. 12, pp. 3964-3969, 1999.

[22] T. Miron and M. Wilchek, "A simplified method for the preparation of succinimidyl carbonate polyethylene glycol for coupling to proteins," Bioconjugate Chemistry, vol. 4, no. 6, pp. 568-569, 1993.

[23] F. Brustolin, F. Goldoni, E. W. Meijer, and N. A. J. M. Sommerdijk, "Highly ordered structures of amphiphilic polythiophenes in aqueous media," Macromolecules, vol. 35, no. 3, pp. 1054-1059, 2002.

[24] J. Teissie and M. Golzio, "Direct imaging of siRNA electrotransfer at the single-cell level," Methods in Molecular Biology, vol. 1121, pp. 121-130, 2014. 

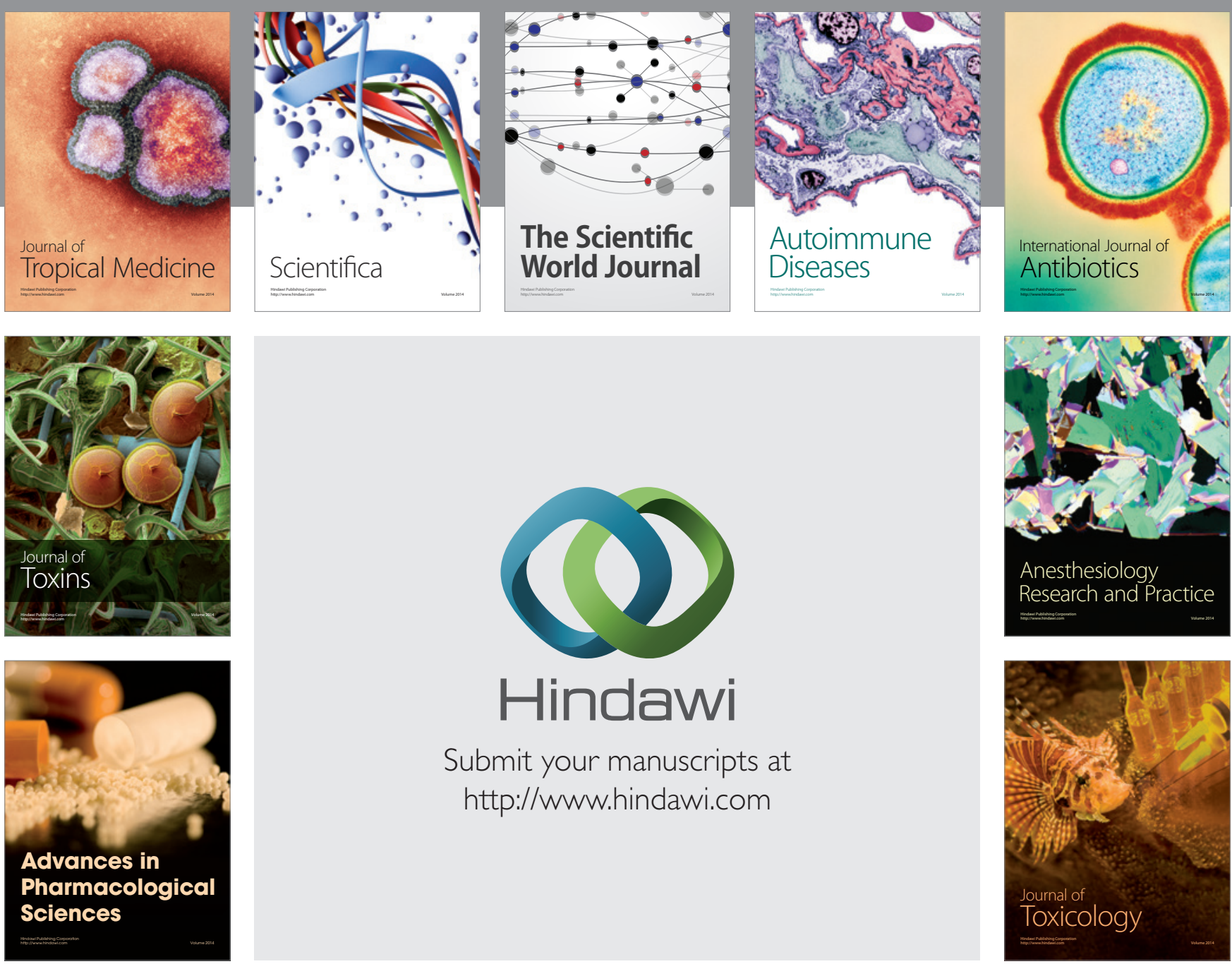

\section{Hindawi}

Submit your manuscripts at

http://www.hindawi.com
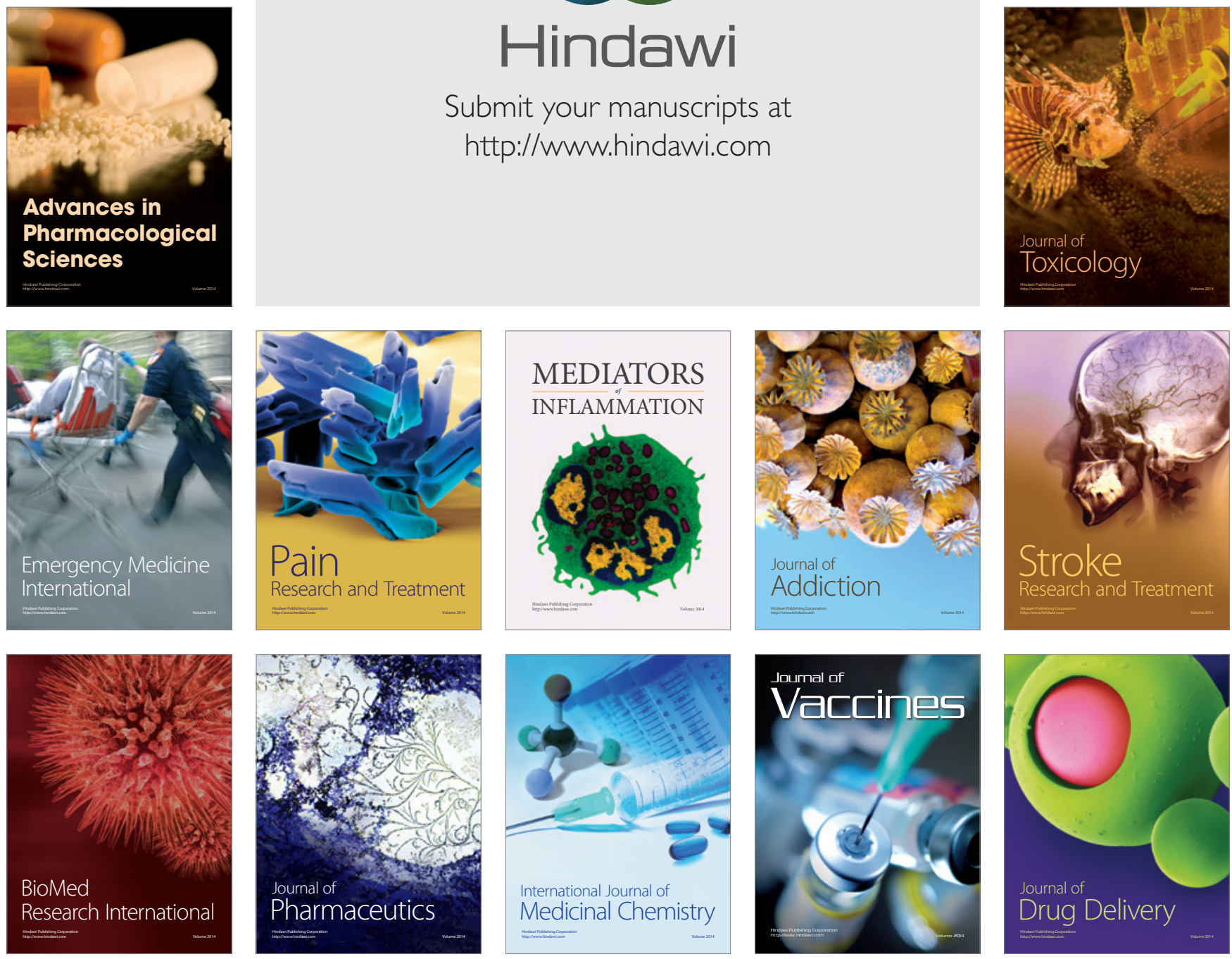